\title{
Capsule Commentary on Kaplan et al., How Have 30-Day Readmission Penalties Affected Racial Disparities in Readmissions?: An Analysis from 2007-2014 in Five US States
}

\author{
Marsha Regenstein, PhD \\ Milken Institute School of Public Health , George Washington University, Washington, D.C., USA.
}

J Gen Intern Med 34(6):1001

DOI: $10.1007 / \mathrm{s} 11606-019-04920-\mathrm{Z}$

() Society of General Internal Medicine 2019

$\mathrm{T}$ he analysis by Kaplan et al. ${ }^{1}$ used inpatient records from Black and White Medicare patients in five states to measure disparities in 30-day readmissions before and after the implementation of the Hospital Readmission Reduction Program (HRRP), an initiative under the Affordable Care Act passed in 2010 with penalties beginning three years later. HRRP substantially broadened financial penalties on individual hospitals with higher-than-expected readmission rates through blunt cuts to Medicare payments. Safety net hospitals, defined as such because they have high numbers of lowincome and publicly insured patients, often suffer from resource constraints yet bear a disproportionate amount of the HRRP penalties. ${ }^{2}$ These hospitals are also characterized by higher numbers of non-white patients, raising concerns that HRRP could lead to greater disparities in readmissions in penalized hospitals.

Kaplan et al. found that Black/White disparities related to readmissions decreased significantly from 2007 to 2010, with the downward trend continuing at a more modest rate postimplementation of HRRP. The finding that nearly all of the drop in disparities occurred in safety net hospitals raises an important red flag about disparate care in non-safety net hospitals, which are less likely to be penalized by HRRP. Nearly the same number of Black patients were represented in the five study states' readmissions in safety net and non-safety net hospitals (about 80,00 versus 75,000), yet readmissionsrelated disparities are virtually eliminated within the safety net group and maintained (or increased over the 2007-2014 period) in the non-safety net group.

The question of whether continued penalties are effective in reducing readmissions further in safety net hospitals is an important one, given the challenges these hospitals face when serving patient populations whose complex social circumstances are not adequately factored into payment rates. ${ }^{3}$ Likewise, we should not lose sight of the disappointing performance of other hospitals that are not making strides toward eliminating disparities in their readmission rates. This study shows that when it comes to readmissions, a rising tide does not lift all boats.

Corresponding Author: Marsha Regenstein, $\mathrm{PhD}$; Milken Institute School of Public Health George Washington University, Washington, D. C., USA (e-mail: marshar@gwu.edu).

\section{Compliance with Ethical Standards:}

Conflict of Interest: The author declares that she does not have a conflict of interest.

Publisher's Note: Springer Nature remains neutral with regard to jurisdictional claims in published maps and institutional affiliations.

\section{REFERENCES}

1. Kaplan CM, Thompson MP, Waters TM. How Have 30-Day Readmission Penalties Affected Racial Disparities in Readmissions?: An Analysis from 2007-2014 in Five US States. J Gen Intern Med. 2019. https://doi.org/10. 1007/s11606-019-04841-x.

2. Joynt KE, Jha AK. Characteristics of hospitals receiving penalties under the Hospital Readmissions Reduction Program. JAMA. 2013; 309(4):342343.

3. Carey K, Lin M-Y. Hospital Readmissions Reduction Program: safety net hospitals show improvement, modifications to penalty formula still needed. Health Affairs. 2016; 35(10):1918-1923. https://doi.org/10.1377/hlthaff. 2016.0537.

Published online March 11, 2019 